\title{
Current Comprehension of the Role of Fatty Acids in the Development of Type 2 Diabetes
}

\author{
Jay Patel and Guoxun Chen* \\ Department of Nutrition, University of Tennessee, Knoxville, Tennessee, USA
}

Received: March 21, 2017; Accepted: April 20, 2017; Published: May 01, 2017

*Corresponding author: Guoxun Chen, Ph.D., Department of Nutrition, University of Tennessee at Knoxville, 229 Jessie Harris Building 1215 West Cumberland Avenue, Knoxville, Tennessee 37996, USA, Tel: 865-974-6257; Fax: 865-974-3491;E-mail: gchen6@utk.edu

\begin{abstract}
Type 2 Diabetes prevalence has increased every year due to a combination of environmental factors and genetics. There has been a gradual realization of the role of fatty acid metabolism in the development of type 2 diabetes. The goal of this review is to summarize the current mechanisms found in research as to the development of insulin resistance relative to disordered fatty acids metabolism. First, we reviewed the definitions, sources, the use and synthesis of fatty acids. Then, we summarized proposed mechanisms by which fatty acid metabolism contributes to the development to type 2 diabetes and insulin resistance. The relationship of fatty acid metabolism and insulin resistance in the liver, adipose tissue, and skeletal muscle was examined in conjunction with the proposed mechanisms. Furthermore, the beneficial roles of polyunsaturated fatty acids in the metabolic diseases and the relationship between poly unsaturated fatty acids and inflammation via cytokines were discussed. In conclusion, altered fatty acid metabolism should be a major focus of research in the future for the prevention and treatment of insulin resistance and type 2 diabetes.
\end{abstract}

Keywords: Insulin Resistance; Fatty Acid Metabolism; Non-insulin Dependent Diabetes Mellitus; PUFAs; Inflammation

\section{Abbreviations}

TNF-a: Tumor Necrosis Factor Alpha; IL: Interleukin; NF-кB: Nuclear Factor Kappa-Light-Chain-Enhancer of Activated B Cells; SOCS: Suppressor of Cytokine Signaling Protein; TLR: TollLike Receptor; JNK: C-Jun N-Terminal Kinases; IRF: Interferon Regulatory Factor; IFN: Interferon; TAG: Triacylglycerol; TNF-R: Tumor Necrosis Factor Receptor; FFA: Free Fatty Acid; ROS: Reactive Oxygen Species; FATP: Fatty Acid Transport Protein; FAT, Fatty Acid Translocase; FABP: Fatty Acid Binding Protein; LCFAC: Long-Chain Fatty Acyl-CoA; DAG: Diacylglycerol; CD36: Cluster of Differentiation 36; VLDL: Very Low Density Lipoprotein; FOXO: Forkhead Box Subgroup 0; PEPCK: Phosphoenolpyruvate Carboxykinase; PC: Pyruvate Carboxylase; PKC: Protein Kinase C; GLUT: Glucose Transporter; G6P: Glucose-6-Phosphate; ATP: Adenosine Triphosphate; PK A/B: Protein Kinase A/B; PUFA: Polyunsaturated Fatty Acids; RA: Retinoic Acid; FA: Fatty Acid; MUFA: Monounsaturated Fatty Acid; CE: Cholesterol Esters; PL: Phospholipids; EPA: Eicosapentaenoic Acid; DHA:
Docosahexaenoic Acid; AMPK: Adenosine MonophosphateActivated Protein Kinase; ACC: Acetyl-CoA Carboxylase; FAS: Fatty Acid Synthase; CPT 1/2: Carnitine Palmitoyltransferase 1/2; cyt C: Cytochrome C;

\section{Introduction}

\section{Types and Sources of Fatty Acids}

At its most basic level, a Fatty Acid (FA) is simply a carboxyl group attached to an aliphatic chain of varying number of carbons and bond types. Due to their characteristics, FAs are classified differently. Based on the number of carbons, FAs are classified as short-chain (C2 to C6), medium-chain (C8 to C14) and long-chain (C16 to longer) FAs. According to the number of double bonds between the aliphatic carbon, FAs are further classified as saturated, monounsaturated (only one double bond) and polyunsaturated (more than one double bond). "Saturated" means that no more hydrogen could be added to the carbons of an FA molecule. The introduction of a double bond creates two configurations, cis (the two carbons on the same side of the double bond) or trans (the two carbons on the opposite side of the double bond). The natural occurring FAs are mainly in the cis configuration whereas food processing introduces trans configuration. The presence of a double between a pair of carbon atoms limits the rotation around the central bond, which results in bending of the FA in cis configuration. The existence of multiple double bonds introduces more complex configurations [1].

Both saturated and unsaturated FAs exist in human foods. Depending on the type, source, and areas of food production, the contents and types of FAs vary dramatically. Saturated fatty acids can be found in many foods we eat such as red meat and dairy products [2]. Cow milk, for example, includes saturated FAs such as butyric acid (also found in butter), myristic acid, and palmitic acid [3]. Common monounsaturated fatty acids, or MUFAs, include palmitoleic acid and oleic acid and can be found in sources such as olive oil and fish. Polyunsaturated fatty acids, or PUFAs, can also be found in fish, dark green leaves like spinach \& kale, nuts, and avocados. Common PUFAs found in marine sources include Eicosapentaenoic Acid (EPA) and Docosahexaenoic Acid (DHA). PUFAs such as EPA and DHA are both omega-3 fatty acids that have been suggested to help combat the risk of cardiovascular 
disease [4]. Saturated fatty acids, however, have been associated with the increased risk of developing metabolic disorders and cardiovascular disease [5].

FAs are not only found in the diet, but also in human and animal cells [6]. They exist in a variety of lipid molecules in the body. Examples include Triacylglycerol (TAG), Monoacylglycerol, Diacylglycerol (DAG), acyl-CoA, Cholesterol Ester (CE), Retinyl Esters, sphingomyelin, cardiolipin, waxes, phospholipids (PLs), etc [7].

\section{Dietary (Exogenous) Lipid Metabolism}

A significant portion of the dietary lipids we ingest is in the form of TAG, which 3 FAs are esterified to the three hydroxyl groups of glycerol [1]. Other common dietary lipids include CE and PLs. Lipid digestion starts in the stomach with the help of gastric and lingual lipases that hydrolyze part of the lipids into DAGs and FAs [8].The churning action of the stomach allows large lipid droplets to be broken into smaller ones, a process that allows further digestion of lipids to become easier. Once the partially digested lipids are moved in the small intestine, they aren't immediately able to be digested by water soluble lipases. Thus, further emulsification in the small intestine occurs with the help of bile acids and salts (e.g., taurocholic acid), which are made from cholesterol in the liver and released from the gall bladder. The bile salts and partially digested lipids form micelles, allowing the binding of colipase (a protein needed for the association of pancreatic lipase with the micelles). TAGs are hydrolyzed to 2-monoacylglycerol and free FAs [9]. After the digestions of enzymes, CEs can be broken down to cholesterol and a FA, and PLs can be broken down into a FA and a lysophospholipid.

The hydrolyzed products from TAGs along with those from CEs and PLs in the micelle are then absorbed by enterocytes. Once in enterocytes TAG, CE and LP are formed again in the smooth endoplasmic reticulum and packed into chylomicrons for transport. Lipoprotein chylomicrons are primarily composed of the dietary lipids TAG and the protein called apolipoprotein B-48. Chylomicrons are transported from the lymphatic system to the blood, and then to adipose and muscle tissues, and eventually taken into the liver in the form of chylomicron remnants [1]. Short- and medium-chain fatty acids can simply be absorbed directly into the blood via the blood capillaries in the intestines [1]. The TAGs are once again hydrolyzed into free FAs and glycerol by lipoprotein lipases associated with the membrane of endothelial cells in the lumen of blood circulation. FAs are then absorbed by adipose tissue and muscle cells and converted back into TAG in the cells for energy storage or use.

\section{Endogenous Lipid Metabolism}

oGram per gram, fatty acids provide more than twice the energy $(\sim 38 \mathrm{~kJ} / \mathrm{g})$ than a carbohydrate or protein molecule [10]. Most energy reserves in humans are stored as TAGs which contain long chain FAs in adipocytes. During postprandial state, glucose is mainly used for power generation and FA biosynthesis. During times of mild activity, vigorous exercise, or fasting, our body starts to use more FAs as an energy source and save glucose for the use in the central nerve system [11]. Normally, insulin inhibits lipolysis in adipose tissues. On the other hand, glucagon stimulates lipolysis, and in turn, increases blood free FA levels [10]. When free FAs enter a cell, an enzyme called acyl-CoA synthetase first converts them into a fatty acyl-CoA in the cytosol [1]. To utilize the stored energy in FAs, they undergo $\beta$-oxidation in mitochondria to generate acetyl-CoA [1]. Short- and mediumchain FAs can enter mitochondria through passive diffusion without any assistance. However, long-chain FAs cannot just enter the mitochondria freely. First, the long-chain fatty acyl-CoA is conjugated to a carnitine molecule by the enzyme Carnitine Palmitoyltransferase I (CPT1) to form a fatty acyl carnitine. This allows for the transport of fatty acyl carnitine cross the outer membrane of the mitochondria. After that, a translocase transfers fatty acyl carnitine across the inner mitochondrial membrane into the mitochondrial matrix. As the fatty acyl carnitine is brought into the matrix, a free carnitine is transported out into the cytosol. Once in the matrix, CPT2 regenerates fatty acylCoA which can then undergo $\beta$-oxidation [1]. The acetyl-CoA in mitochondria can be either completely oxidized into $\mathrm{CO} 2$ or converted to ketone bodies (acetone, $\beta$-hydroxybutyrate, and acetoacetate) in hepatocytes [9]. Due to the presence of double bonds in unsaturated FAs additional enzymes such as 2,4 dienoylCoA reductase and enoyl-CoA isomerase are needed to rearrange the double bond positions for the FAs to get into the $\beta$-oxidation process. As the first step of $\beta$-oxidation of a saturated FA is to introduce a double bond and an FADH, the complete oxidation of an unsaturated FA produces less ATP than a saturated one with equal carbon numbers [10].

\section{Fatty Acid Biosynthesis}

Excessive glucose and $\alpha$-keto acids of amino acids in cells can be converted into FAs for storage. This is achieved through de novo lipogenesis. The main precursor of FA formation is acetyl-CoA, which can be generated from FA $\beta$-oxidation, from the conversion of pyruvate to acetyl CoA, or from amino acid metabolism. Pyruvate from glycolysis or other sources is first converted into acetyl $\mathrm{CoA}$ in mitochondria by pyruvate dehydrogenase complex. Acetyl CoA is transported out from mitochondria into the cytosol in the form of citrate. Citrate is hydrolyzed again into oxaloacetate and acetyl-CoA by the enzyme ATP citrate lyase again. The cytosolic acetyl-CoA is condensed with a $\mathrm{CO} 2$ to generate malonyl-CoA by Acetyl-CoA Carboxylase (ACC). As ACC allows for the committed step to produce malonyl$\mathrm{CoA}$, it is strongly regulated by levels of insulin, glucagon, and FA levels in the cells [1]. The hormones glucagon and epinephrine can activate a signal transduction pathway in the cell to activate AMP-Activated Protein Kinase (AMPK) which can phosphorylate ACC, deactivating it [1]. Additional deactivating factors include long/short fatty acyl-CoA chains and Protein Kinase A (PKA) [12]. On the other hand, insulin can activate protein phosphatase 2A to dephosphorylate ACC, reactivating it [13]. In addition, insulin can activate phosphodiesterases that can convert cAMP to 5'AMP, decreasing the activity of PKA and AMPK [1]. If ACC is phosphorylated, it can be partially activated by the binding of citrate molecules coming from the mitochondrial matrix to the 
cytosol.

Acetyl CoA and malonyl CoA are building blocks for de novo FA biosynthesis and used by Fatty Acid Synthases (FAS). Mammalian FAS is one long polypeptide chain with all the domains required for FA synthesis. FA synthesis occurs in the cytoplasm. Intermediates in FA synthesis link to the -SH group of an Acyl Carrier Protein (ACP). The FA chain can grow longer with the addition of two-carbon units from malonyl-ACP (Acetyl CoA + ATP + HCO3-). As FA synthesis proceeds, NADPH will be primarily used for reduction. The NADPH can come from two sources: the pentose phosphate pathway or during the process of bringing out carbon precursors to the cytosol when malate undergoes decarboxylation to pyruvate and NADP+ is reduced to NADPH. NADPH is primarily used in two reduction steps in FA biosynthesis. $\beta$-ketoacyl-ACP is reduced to D-3-hydroxyacylACP via $\beta$-ketoacyl-ACP reductase; enoyl-ACP reductase reduces trans-2-enoyl-ACP to butyryl-ACP; both enzymes can reduce their respective substrates using NADPH [1]. After the synthesis of FAs, they can be esterified into TAG and transported into adipose tissue for storage.

From here, palmitate can be further elongated, or it undergoes desaturation to form unsaturated FAs [1]. Elongation of a FA will continue until the FAS produces the 16-carbon long palmitate [10]. Elongation of palmitate to longer saturated fatty acids occurs in the smooth endoplasmic reticulum via enzymes called elongases. Malonyl-CoA provides two carbons to be added on to the fatty acyl-CoA. Elongases are encoded from Evolv (Elongation-of-Very-Long-Chain-Fatty Acids) genes and are involved in the condensation step of the elongation process [14]. There have been about seven elongase enzymes identified (ELOVL 1-7) [15]. These elongase enzymes help to make very-long-chain fatty acids that are essential components of sphingolipids or wax esters [14]. Currently, it is thought that ELOVL 1,3, and 6 are involved in elongation of monounsaturated VLCFAs; ELOVL 2, 4, and 5 participate in elongating PUFAs [14]. Desaturation to form unsaturated fatty acids is done by enzymes called fatty acyl-CoA desaturases, which create double bonds at specific locations of FAs. Stearate (18:0) is elongated from palmitate, and its desaturation to oleate is done by stearoyl-CoA desaturase. Similarly, palmitate can be desaturated to palmitoleate [10]. Unlike plants, mammals can't form double bonds at carbon 10 and above counted from the carboxylic end due to the lack of the desaturases that can catalyze the reactions. Hence, essential FAs such as linoleate and linolenate must be acquired from external sources [1]. Plants have these desaturases in the chloroplast and the endoplasmic reticulum. Once these essential FAs are ingested, humans can elongate or desaturate them to form things such as eicosatrienoic, and arachidonate by using $\Delta 5$ and $\Delta 6$ desaturases [16].

Current theories of effects of fatty acid metabolism on the development of non-insulin dependent diabetes mellitus

\section{Types of diabetes}

Diabetes is the inability of the body to either produce or respond to insulin and eventually lead to high blood glucose level and a host of bodily disorders such as heart disease, renal failure, and retinopathy [17]. There are mainly two types of diabetes. Type 1 diabetes (also known as insulin-dependent diabetes, IDDM) and type 2 diabetes (also known as non-insulin dependent diabetes mellitus, NIDDM). Both are characterized by an eventual loss of pancreatic $\beta$-cells [18]. Hyperglycemia has been considered the primary consequence of diabetes due to work done by Oskar Minkowski [19]. Minkowski was hypothesized to have tasted the urine of dogs that had undergone a pancreatectomy after noticing many flies hovering around the urine; he thought that a missing pancreatic enzyme might have caused glycosuria and hyperglycemia. With later studies, it was found that insulin is a major hormone produced by $\beta$-cells of the pancreas. They have different pathogenic mechanisms.

IDDM is an autoimmune disorder that eventually leads to the destruction of $\beta$-cells. Factors include viruses, genetic predisposition, or elevated levels of glucose $[20,21]$. One mechanism behind IDDM is thought to arise from immune cells producing cytokines (e.g., IL-1 $\beta$, tumor necrosis factor (TNF)- $\alpha$, and interferon (IFN)- $\gamma$ ) that act on transcription factors such as NF- $\kappa$ B to induce or impede $\beta$-cell genes involved in the cell functions and survival. The IL-1 $\beta$ pathway, for example, can lead to increased expression levels of genes Bid and Bak, which trigger mitochondria to release cytochrome $C$ (cyt $C$ ). In addition, this pathway can increase expression levels of iNOS gene which increases nitric oxide production in the cell, activating p53 and subsequent mitochondrial release of the "death signal" cyt C [18]. The IFN- $\gamma$ pathway increases transcription of the Caspace- 3 gene that makes a caspace-3 protein that leads to apoptosis in part due to activation help from cyt C [18]. Transcription factor activation can lead to increased nitric oxide, greater chemokine production, and emptying the ER of calcium, causing ER stress [18]. MAPK becomes activated and causes mitochondria to release apoptosis signals, resulting in B-cell destruction.

The mechanism behind the occurrence of NIDDM and $\beta$-cell destruction is more complex and thought to be due to a multitude of factors $[18,20,21]$. Studies have now shown that several mechanisms can lead to insulin resistance [22].

\section{Current theories on how fatty acid metabolism is in- volved in insulin resistance}

For a long time, research emphasis of diabetes was placed on studying the glucose-insulin axis. In 1992, JD McGarry emphasized again that disordered fatty acid metabolism could be a primary cause or at least contributing to insulin resistance [19]. This occurs in both IDDM and NIDDM [19, 21-23].

To start off, we'll examine one of the earliest observation that suggested a relationship between fatty acid metabolism and glucose metabolism in the skeletal muscle observed by Philip Randle, the Randle cycle. The Randle cycle, also known as the glucose-fatty acid cycle in the muscle, is a phenomenon suggesting that glucose and fatty acids compete with each to be used as a source for energy production. 


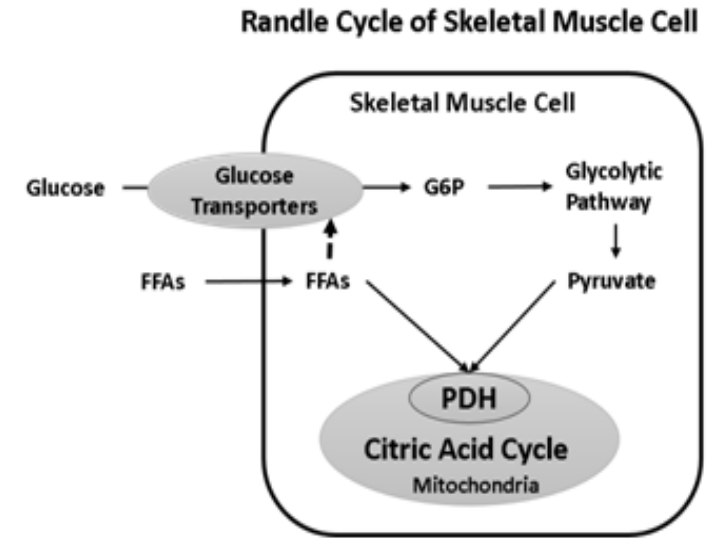

Figure 1: Randle Cycle of Skeletal Muscle Cell

Normally, glucose enters the skeletal muscle through glucose transporters, goes through glycolysis to be made into pyruvate. Pyruvate is converted to acetyl-CoA via PDH for the entry in the citric acid cycle. During the fasting state, the plasma FFA concentration rises. The FFAs can enter the citric acid cycle in the mitochondria to generate ATP for cell processes. The utilization of FFAs in the muscle cells interferes with the entry of glucose into the cells and the conversion of pyruvate into acetyl CoA through the PDH complex. This leads to the reduction of glucose usage in the skeletal muscle, a glucose "sparing' effect, and more glucose is available for neuronal cells. FFA: Free Fatty Acid; G6P: Glucose6-Phosphate; PDH: Pyruvate Dehydrogenase.

Figure 1 shows the interaction between glucose and fatty acid usage in the Randle Cycle. This has been used to explain the glucose sparing effect during the fasting state. In the fed state, abundant supply of glucose leads to its use as the primary source of energy. This is associated with a greater insulin to glucagon ratio, which stimulates glucose uptake and fatty acid esterification. During times of fasting or starvation, there would be increased mobilization of FAs from adipocytes, increasing blood concentrations of FFAs [1].

Figure 2 describes how FAs are used during the fed and fasting states. Both liver and muscle cells oxidize FA through $\beta$-oxidation. In the liver, excessive $\beta$-oxidation leads to accumulation of acetyl-CoA and conversion of acetyl-CoA to ketone bodies. These ketone bodies were soluble in blood and can cross the blood-brain barrier, allowing them to be taken up by the brain, heart, and kidney cells and converted back to acetyl-CoA for energy production [10]. During fasting, pyruvate dehydrogenase complex activity would be reduced, glucose oxidation decreases, intracellular citrate concentration increases, phosphofructokinase is inhibited, Glucose-6-Phosphate (G6P) concentration is increased, resulting in hexokinase inhibition, ultimately leading to decreased glucose uptake by muscle. The elevation of gluconeogenesis in the liver and the reduction of glucose utilization in the muscle result in the maintenance of blood glucose level in a normal range.

This mechanism would allow glucose to be "spared" (glucose is saved for use in other cells) per the Randle hypothesis and lead FAs being oxidized for energy [24]. This hypothesis provided a plausible mechanism as fatty acid oxidation reduces

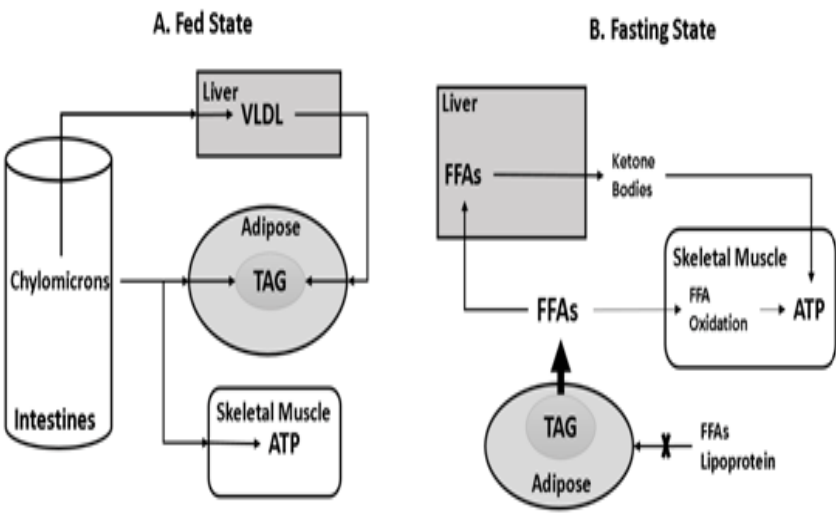

Figure 2: Processing of FFAs in the Fed (A) and Fasting (B) States

A) From the small intestines, absorbed FFAs are esterified and packed into chylomicrons for the transport to other parts of the body. Lipids in chylomicron remnants are taken and converted to VLDL in the liver. The FFAs in TAG of VLDL and chylomicrons will be taken into the adipose tissue and stored as TAG. FFAs in skeletal muscle can also be oxidized for the production of ATP. Cells' energy needs are mostly fulfilled by glucose oxidation in the fed state. B) During the fasting state, FFAs are derived from TAG lipolysis in the adipocytes. The elevated oxidation of FFAs caused the induction of ketone bodies. These ketone bodies from the liver and FFAs from lipolysis can be utilized in the skeletal muscle for the production of ATP. ATP: Adenosine Triphosphate; TAG: Triacylglycerol; FFA: Free Fatty Acids; VLDL: Very Low Density Lipoprotein.

glucose utilization.

Based on the Randle Cycle, an increase of FA availability will cause inhibition of the glycolytic pathway through the pyruvate dehydrogenase complex and glucose uptake in the muscle cells [15]. Other studies have suggested that the increase of FA levels is responsible for insulin resistance, which leads to reductions in glucose uptake, glucose usage, glycogen synthesis, and glucose6-phosphate levels without affecting pyruvate dehydrogenase complex $[25,26]$. One possible mechanism of lowered glucose6-phosphate could be inhibition of hexokinase II which is directly regulated by insulin. This shows that there must be other plausible mechanisms that are not accounted for in the Randle Cycle that could elicit insulin resistance. Research showed that high glucose and insulin concentrations found by Randle could inhibit fatty acid oxidation via the inactivation of CPT-1 at the mitochondrial outer membrane [27].

Studies have also shown that insulin resistance is more of a consequence of impaired insulin signal transduction, which lead to the impairment of translocation of the insulin-regulated glucose transporter 4 (GLUT4) [28-30]. In addition, the increased TAG and DAG levels in skeletal muscle cells were found to impair GLUT4 translocation [31]. The proposed mechanism is that the increases in TAG metabolites such as DAG or fatty acyl-CoA activate a Ser/Thr protein kinase cascade initiated by protein kinase C, leading to phosphorylation of the Ser/Thr binding sites on the insulin receptor substrates (IRS) $1 \& 2$. This decreases the possibility of PI 3-kinase activation; therefore, glucose transport into the cell is diminished even when insulin binds to its receptor [30, 32]. 
Potential Insulin Resistance Mechanism in Skeletal Muscle Cells

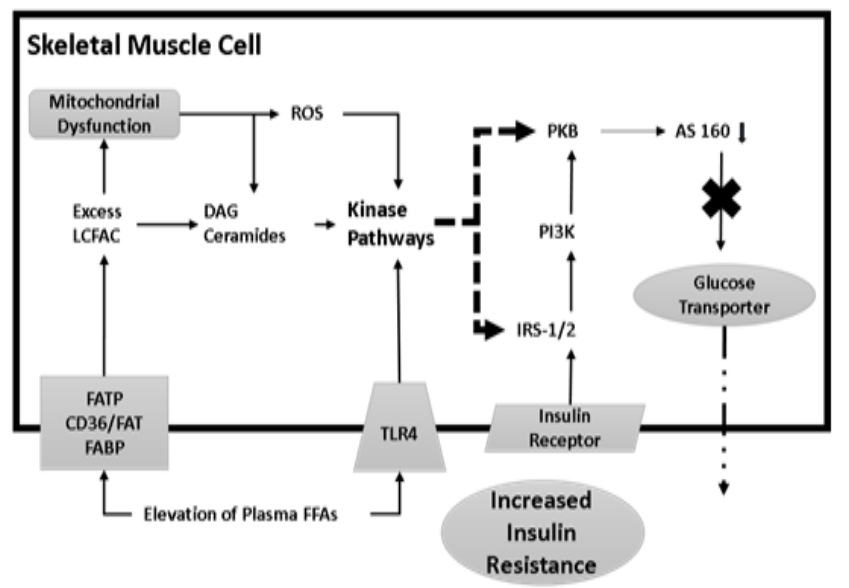

Figure 3: Potential Insulin Resistance Mechanism in Skeletal Muscle Cells

Plasma FFAs and cytokines affect the functions of the skeletal muscle cells. The excessive entry of FFAs into the muscle cells causes the elevation of their oxidation in mitochondria, which increases the production of ROS. These ROS are thought to damage the mitochondria and result in mitochondrial dysfunctions. The mitochondrial dysfunctions prevent proper oxidation of FFA, resulting in increased intracellular levels of DAG and ceramides. The DAG, ceramides, and ROS converge with pathways of TLR 4 activated by FFAs and can lead to inhibition of activities of IRS $1 / 2$ and PKB. This results in inhibition of the insulin receptor signaling pathway and decreased translocation of glucose transporter 4 to the cell membrane, which leads to reduction of glucose uptake and longer glucosemia. FATP: Fatty Acid Transport Protein; FAT: Fatty Acid Translocase; FABP: Fatty Acid Binding Protein; LCFAC: Long-Chain Fatty acylCoA; DAG: Diacylglycerol; TAG: Triacylglycerol; IRS 1/2: Insulin Receptor Substrate 1/2; PI3K: Phosphatidylinositide 3-Kinase; PKB: Protein Kinase B; ROS: Reactive Oxygen Species; TLR4: Toll-Like Receptor 4; FFA: Free Fatty Acid; CD36: Cluster of Differentiation 36.

insulin receptor substrates (IRS) $1 \& 2$. This decreases the possibility of PI 3-kinase activation; therefore, glucose transport into the cell is diminished even when insulin binds to its receptor [30, 32].

This mechanism is demonstrated in Figure 3 for a skeletal muscle cell.

As shown in Figure 4, JD McGarry postulated a mechanism that would expand on the Randle hypothesis for the development of NIDDM. He proposed that in response to overeating or over-nutrition, the pancreas secretes more insulin to regulate glucose homeostasis. Insulin is also needed for hepatic lipogenesis. Therefore, the elevated blood insulin stimulates the increasing lipogenesis in the liver, resulting in excess secretion of Very Low-Density Lipoprotein (VLDL). This leads to the storage of TAG in the adipose tissue in the earlier and healthy state, and accumulation of TAG in the muscle in the later and insulin-resistant state. Increased TAG accumulation in cells other than the adipocytes will interfere with glucose oxidation, which leads to mild insulin resistance. The spilled over glucose (higher blood glucose levels) stimulates more insulin secretion from $\beta$ cells and results
JD McGarry Theory of Whole Body Insulin Resistance

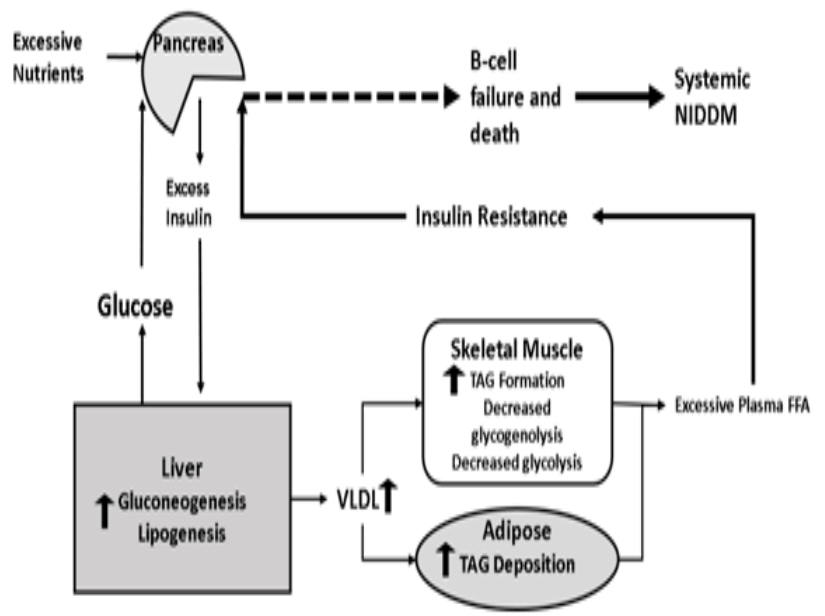

Figure 4: JD McGarry Theory of Whole Body Insulin Resistance During the early phases of insulin resistance, excess intake of nutrients stimulates more insulin secretion. This extra insulin production results in the liver producing more VLDL that can build up in skeletal muscle and the adipocyte. The adipocyte experiences hypertrophy and results in increased TAG lipolysis to increase plasma FFA levels that can then go back to the pancreas to stimulate even more insulin secretion. In the later phases of insulin resistance, the liver maximizes its production of VLDL, and there is elevations of 1 lipolysis in skeletal muscle and adipocytes. At the same time, there is an increase of hepatic gluconeogenesis. This in combination with the excessive plasma FFAs leads to over stimulation of the pancreas. On the other hand, excessive plasma FFAs will cause insulin resistance in tissues and organs, which establishes a vicious cycle and will eventually lead to $\beta$-cell failure and development of overt NIDDM. VLDL: Very Low Density Lipoprotein; TAG: Triacylglycerol; FFA: Free Fatty Acids; NIDDM: Non-Insulin Dependent Diabetes Mellitus.

in hyperinsulinemia. Hyperinsulinemia will further stimulate the hepatic lipogenesis and cause the elevation of plasma free fatty acid levels due to the limited places to store the excessive fat. This creates a vicious cycle. With the development of hepatic insulin resistance, insulin no longer suppresses the hepatic gluconeogenesis, but still stimulates lipogenesis. Eventually, the pancreatic $\beta$-cells could not produce more insulin to compensate for the increased blood glucose levels, and overt diabetes occurs [19, 22]. This shows that glucose and fatty acid metabolism may be able to impact each other.

Another possible mechanism is that increased FA levels prevent insulin action of glycogenolysis, which results in glycogen being broken down and increasing blood glucose levels [33]. This disordered FA metabolism can cause the liver to overproduce glucose. Another theory to this is that DAG made in the liver cell activates $\mathrm{PKC} \varepsilon$, which phosphorylates the tyrosine kinase portion of the insulin receptor, decreasing insulin activity [34]. This can lead to less AKT2 activation, which then decreases the number of phosphorylated glycogen synthase kinase 3 (GSK3), enhancing its activity and decreasing glycogen synthesis by phosphorylat- 
ing glycogen synthase [35]. Reduced AKT2 activation results in less transcriptional factors called forkhead box protein $\mathrm{O}$ (FOXO) from being phosphorylated, in which leads to transcription of genes that aid in gluconeogenesis such as phosphoenolpyruvate carboxylase (PEPCK) and glucose-6-phosphatase [36]. This then results in increased hepatic gluconeogenesis as shown in Figure 5.
As shown in Figure 6, a similar feature in both liver and skeletal muscle oxidation pathways is the use of Ser/Thr kinases that phosphorylate the insulin receptor, conferring insulin resistance. DAG activates the Ser/Thr kinase PKCE, which inactivates the insulin receptor. DAG can be synthesized from FA re-esterification or de novo lipogenesis [35]

\section{Potential Insulin Resistance Mechanism in Hepatocyte}

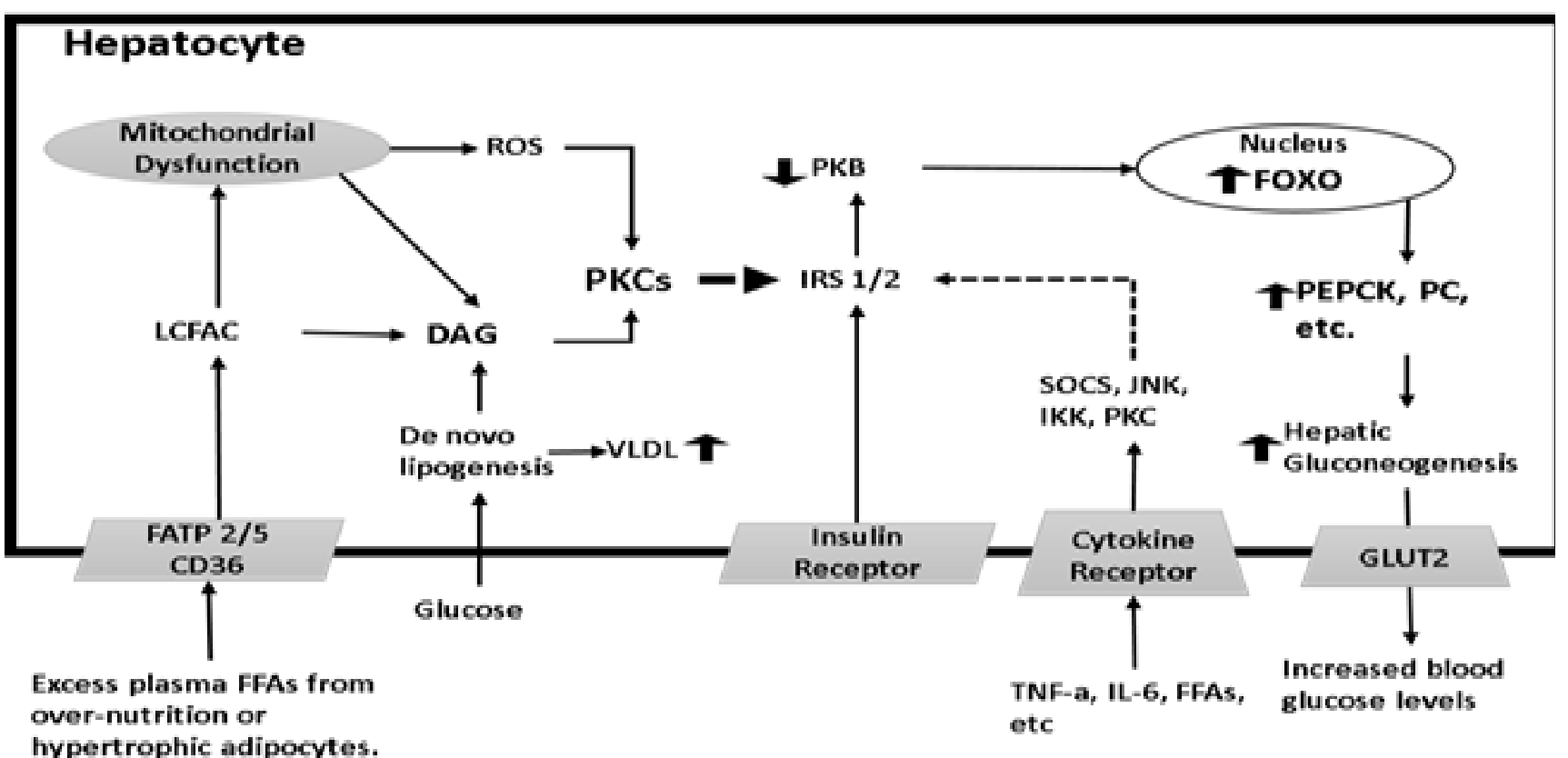

Figure 5: Potential Insulin Resistance Mechanism in Hepatocytes

In the liver, the entry of FFAs into the hepatocytes leads to their oxidation. The excessive oxidation in the mitochondria enhances the production of ROS, which results in the elevation of intracellular DAG level. Glucose can be used to generate DAG and VLDL via de novo lipogenesis. With the rise of DAG levels, there is an increase of activation of PKCs, which can inhibit activities of IRS 1 and 2. In addition, the binding of cytokines to their cytokine receptor can activate several kinase pathways, such as JNK, IKK, and PKCs, which also inhibit the activities of IRS 1 and 2 proteins and will in turn reduce the insulin receptor signaling pathway. This leads to less phosphorylated AKT2 and FOXO. FOXO not being phosphorylated results in increased production of gluconeogenic enzymes that results in an exaggerated production and release of glucose into blood. FATP 2/5: Fatty Acid Transport Protein 2/5; LCFAC: Long-Chain Fatty Acyl-CoA; FFA: Free Fatty Acids; DAG: Diacylglycerol; VLDL: Very Low Density Lipoprotein; FOXO: Forkhead Box Subgroup 0; PEPCK: Phosphoenolpyruvate Carboxykinase; PC: Pyruvate Carboxylase; PKC: Protein Kinase C; GLUT 2: Glucose Transporter 2; SOCS: Suppressor of Cytokine Signaling Protein; IKK: Ikb Kinase; JNK: c-Jun N-Terminal Kinases; TNF-a: Tumor Necrosis Factor Alpha; IL 6: Interleukin 6; IRS 1/2: Insulin Receptor Substrate 1/2; PKB: Protein Kinase B; CD36: Cluster of Differentiation 36.

When the hepatocytes or skeletal muscle cells receive an increase of FAs from the plasma, DAG synthesis from FA can increase due to the capacity of mitochondria to oxidize fatty acyl CoAs and impairment of mitochondrial functions [37]. Longchain Fatty Acids (LCFAs) normally enter the cell through transport proteins. The skeletal muscle cells use fatty acid translocase (FAT/CD36) or fatty acid transport protein (FATP) 1 \& 4.

The liver can use transport proteins such as FATP2,3 and 5 and CD 36 [38]. When an excess of FAs are taken into the cells and go to the mitochondria, FAs undergo $\beta$-oxidation, result- ing in the generation of an increased number of Reactive Oxygen Species (ROS) when the acetyl-CoA from $\beta$-oxidation goes to the citric acid cycle and when the NADH and FADH2 goes to the electron transport chain [34].

This will eventually result in impaired $\beta$-oxidation, decreased insulin response, decreased mitochondrial gene expression (e.g., the genes needed to make cytochrome $\mathrm{c}$ and Complex I and III), and increased FFA derivatives (e.g., ceramides and DAG) being present in the cell. 
The Effects of FFAs on Hepatocytes and Skeletal Muscle Cells

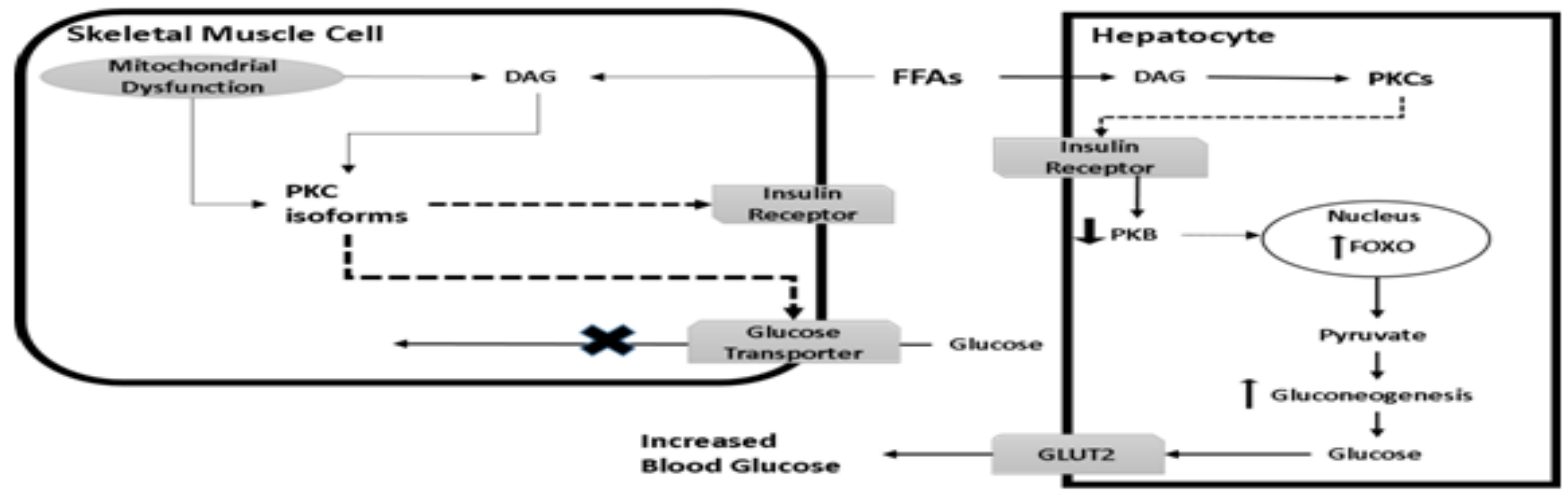

Figure 6: The Effects of FFAs on Hepatocytes and Skeletal Muscle Cells

FFAs can have a dual impact on the liver and skeletal muscle. FFAs entering the skeletal muscle are converted to DAG due to mitochondria dysfunction. DAG and ROS from the mitochondria can activate PKCs. This results in inhibition of the insulin receptor signaling and glucose uptake in the muscle cells. In hepatocytes, DAG from FFA utilization can activate PKCs, leading to impairment of the insulin receptor signaling pathway. In addition, less inhibition of the FOXO protein increases the production of gluconeogenic enzymes such as PEPCK and PC, leading to gluconeogenesis and elevation of blood glucose level. FFA: Free Fatty Acids; DAG: Diacylglycerol; VLDL: Very Low Density Lipoprotein; FOXO: Forkhead Box Subgroup O; PKC: Protein Kinase C; GLUT: Glucose Transporter; PKB: Protein Kinase B.

Several of these derivatives and ROS (oxidative stress) can in turn activate several stress-induced, Ser/Thr kinases such as JNK, NF$\kappa \mathrm{B}$, and multiple forms of PKC that can phosphorylate IRS1 at the serine residue and decrease phosphorylation at the tyrosine residue, reduce the signal from the insulin receptor to IRS-1 [37].

\section{Role of adipose tissue in insulin resistance}

Insulin resistance is a common feature of human obesity. Obesity results in fat accumulation in various parts of the body. Many studies have shown strong correlations between obesity (fat accumulation) and NIDDM as nearly 90\% people with NIDDM are obese [39]. The location of the fat depots has also been suggested to act differently in insulin resistance [23]. In the human body, the majority of fat $(\sim 80 \%)$ is stored in subcutaneous adipose tissue, and $\sim 10 \%$ is stored in visceral adipose tissue [40]. FA mobilization occurs the fastest at visceral adipocytes and relatively slower in subcutaneous adipocytes [23]. The effects of FA flux from visceral fat on insulin action in the liver have been proposed. It has been thought that an increased FA concentrations in the hepatic portal vein, resulting in increased gluconeogenesis, increased TAG synthesis, and decreased insulin clearance or removal from the body. FFA mobilized from subcutaneous fat results in increased FA concentrations for the pancreas and skeletal muscle, resulting in less insulin release by the pancreas and decreased glucose utilization by muscle [40]. Increased visceral and subcutaneous fat in the abdominal regions has been associated with increased risk of metabolic disorders like NIDDM and cardiovascular problems [41].

As one increases caloric intake, subcutaneous adipocytes hypertrophy from excess fat deposition, resulting in activation of pathways such as the nuclear factor-KB pathway. Hypertrophy of the adipocyte causes the cell to release a protein called Monocyte Chemoattractant Protein-1 (MCP-1) that attracts mac-
Potential Insulin Resistance Mechanism in Adipocyte

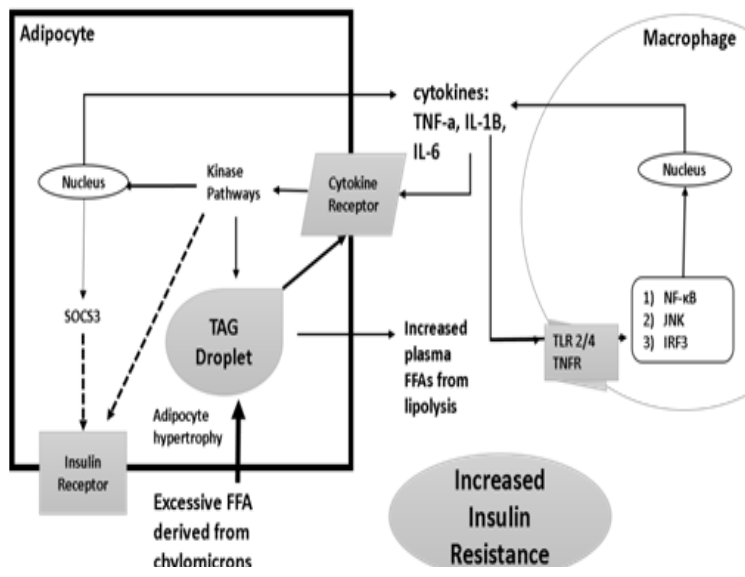

Figure 7: Potential Insulin Resistance Mechanism in Adipocytes Increases of fat from absorption and de novo lipogenesis result in the adipocyte hypertrophy and the elevation of plasma levels of FFAs. Next to the adipocyte, cytokines and FFAs lead to more production of pro-inflammatory cytokines from immune cells such as a macrophage through the activations of pathways such as NF-kB, JNK and IRF3 after interacting with receptors such as TLR2/4 and TNFR. In the adipocyte, cytokines activate several kinase pathways that directly and indirectly such as increasing the amount of SOCS3 inhibit the insulin receptor activity, increase lipolysis of TAG, and cytokine secretion. Eventually, insulin resistance occurs in adipose tissue. TNF-a, tumor necrosis factor alpha; IL 6/1B: Interleukin 6/1B; NF- $\mathrm{BB}$ : Nuclear Factor Kappa-Light-ChainEnhancer of Activated B Cells; SOCS3: Suppressor Of Cytokine Signaling Protein 3; TLR2/4: Toll-Like Receptor 2/4; JNK: c-Jun N-Terminal Kinases; IRF3: Interferon Regulatory factor 3; TAG: Triacylglycerol; TNF-R: Tumor Necrosis Factor Receptor; FFA: Free Fatty Acid. 
rophage molecules to enter the adipocyte. Macrophages at the site will increase the release of cytokines, causing the adipocyte to increase TAG lipolysis, increasing insulin resistance, and generate even more pro-inflammatory cytokines that act in a positive feedback loop [42]. Figure 7 illustrates this interplay between immune cells, adipocytes, and cytokines.

\section{Benefits of PUFAs in insulin sensitivity}

What is the role of unsaturated fatty acids on development of insulin resistance? Research data have shown that treatment of FA to cells and feeding animals with high-fat diets will cause the development of insulin resistance. Interestingly, plenty of data indicated that diets containing a high content of PUFA could increase insulin sensitivity. There are two main types of PUFAs: omega-3 (n-3) and omega-6 (n-6). N-3 include FAs such as DHA and EPA (in fish) and $\alpha$-Linolenic acid (ALA) (in avocados and flaxseed oil); N-6 includes compounds such as linoleic acid (in safflower oil and avocados) [43]. In general, unsaturated fats have been found to improve insulin resistance [44]. In a metaanalysis that looked at 102 trials and 4,660 human participants, it was found that unsaturated fatty acids, such as PUFAs and MUFAs can significantly help control blood glucose levels. MUFAs such as oleic acid can aid in improving blood glucose levels and insulin sensitivity. More profoundly, PUFAs, primarily linoleic acid, have been found to help in the long-term regulation of blood glucose levels, improve insulin sensitivity, and increase insulin secretion ability to a stronger degree than MUFAS acids [32]. While MUFAs have been associated with improved insulin sensitivity [45], other studies have found a positive relationship between MUFAs consumption and development of NIDDM; however, this may be due to the different sources of the MUFAs (e.g., avocado vs. red meat) [46]. In addition to improving insulin sensitivity, PUFAs have been found to decrease the incidence of cardiovascular disease among people such as the Eskimos with their rich marine diet [47].

Studies in rats have shown that saturated fatty acids aid in developing insulin resistance whereas PUFAs aid in improving insulin sensitivity by converting fats into TAG to decrease circulating levels of FAs [48]. Rats fed a rich n-3 PUFA diet resulted in increasing lipid oxidation, decreasing the number of reactive oxidative species generated in the mitochondria, and decreasing the number of pro-inflammatory cytokines (e.g., TNF $\alpha$ andMCP-1) [49]. Figure 8 illustrates how the interplay between PUFAs and cytokines can mediate inflammation and developing insulin resistance.

The Combined View of PUFA and Cytokines in the Alteration of Insulin Signaling

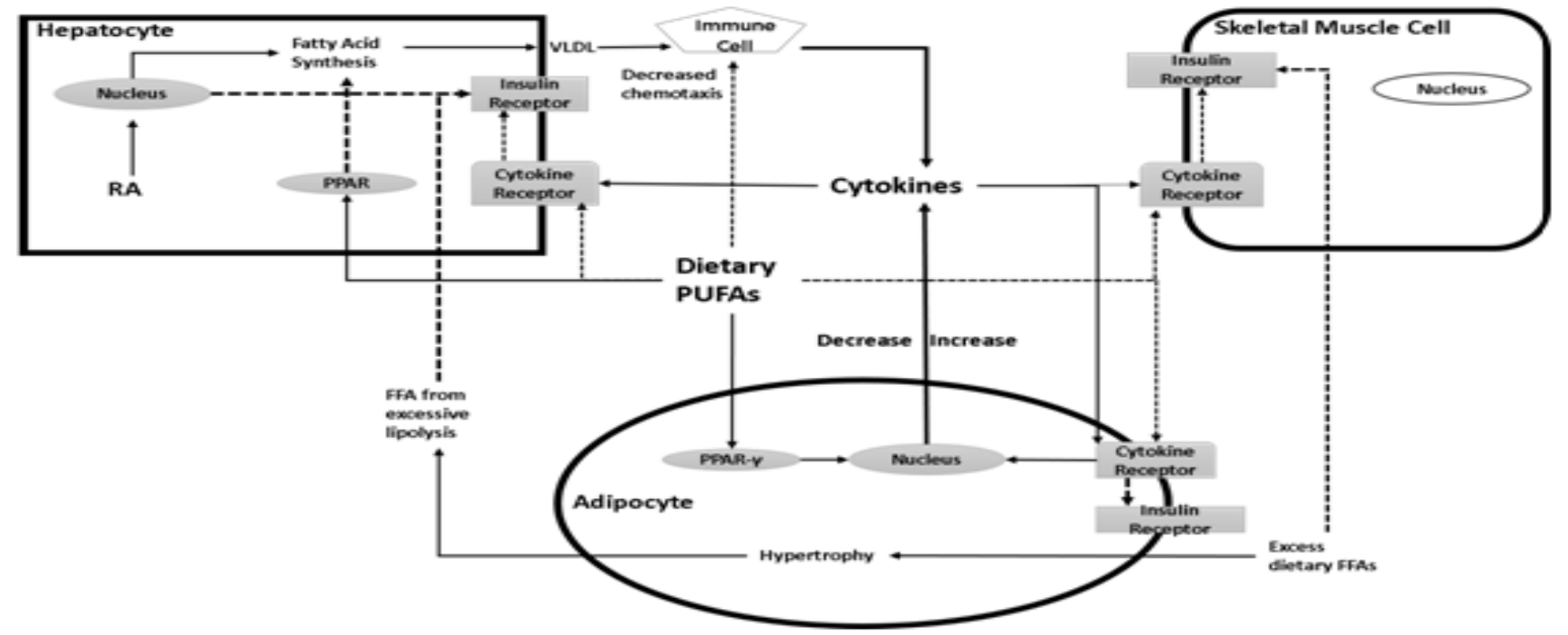

Figure 8: The Combined View of PUFA and Cytokines in the Alteration of Insulin Signaling

The excessive intake of dietary lipids and de novo synthesis of FFAs will be stored in adipose tissue, which leads to adipocyte hypertrophy. Additional intake of micronutrients such as vitamin A can also facilitate lipogenesis. For example, Retinoic Acid (RA), a metabolite of vitamin A, may increase FA synthesis in the liver. FFAs binding to receptors on the immune cell can stimulate release of pro-inflammatory cytokines, which in turn stimulates additional release of cytokines from both the adipocyte and other immune cells, creating a positive feedback loop. These cytokines can then activate cytokine receptors on the skeletal muscle, liver, and adipose and lead to the inhibition of the insulin receptor signaling pathways via activation of several kinase pathways. On the other hand, dietary PUFA, especially n-3 PUFAs, can possible counteract the impact of cytokines and FFAs on insulin resistance. Dietary PUFAs can activate PPARs in the adipocyte and liver to decrease production of cytokine and inhibition of FA synthesis, respectively. PUFA can also inhibit the activation of cytokine receptors in skeletal muscle cells, adipocytes, hepatocytes and immune cells via alteration of FA composition in membrane phospholipids. This results in decreased inflammatory impact from the cytokines and decreased whole-body insulin resistance. VLDL: Very Low Density Lipoprotein; FA: Fatty Acid; PPAR y:, Peroxisome Proliferator-Activated Receptors y; PUFA: Polyunsaturated Fatty Acids; FFA: Free Fatty Acids; RA: Retinoic Acid. 
Several human and animal studies have shown that both types of PUFAs don't promote inflammation like that done by saturated fatty acids; rather, PUFAs may play a role in inhibiting inflammation and improving insulin sensitivity [47, 50, 51]. Research has found that high, saturated fat diets increase the translocation of the endotoxin Lipopolysaccharide (LPS) to the blood to elicit a strong inflammatory response from immune cells [52]. As shown in human studies, PUFAs, especially n-3 ones, could possibly counteract this via decreasing immune cell chemotaxis and production of inflammatory cytokines [50]. Another possible mechanism is through binding of PUFAs to the peroxisome Proliferator-Activated Receptor (PPAR)- alpha [53]. In studies done with leptin-deficient $o b / o b$ mice, PUFAs resulted in repressing the sterol regulatory element binding protein-1, decreasing the production of proteins such as fatty acid synthase, and binding to PPAR-alpha receptor, increasing expression of acyl-CoA oxidase and CPT-1, resulting in decreased hepatic steatosis and decreased insulin resistance [53]. PUFAs can change the membrane composition of the cells involved the inflammatory process, resulting in inactivation of the cytokine receptor, decreased production of cytokines, and inhibiting production of inflammatory eicosanoids from arachidonic acid [54].

While consuming both $n-3$ and n-6 PUFAs is necessary for the body, consuming a proper ratio of PUFAs is important to decrease inflammatory disease processes. n-6 PUFA consumption normally greatly exceeds consumption of $n-3$ PUFAs in Western diets [55]. The n-6 PUFA Arachidonic Acid (AA) can lead to the formation of pro-inflammatory eicosanoids such as prostaglandins, leading to increased production of cytokines from inflammatory cells [50]. The n-3 PUFAs act as anti-inflammatory agents by acting as AA antagonists, decreasing the amount of AA in the cells and the amount of prostaglandins produced. In turn, there are less inflammatory cytokines such as IL-6 and TNF- $\alpha$ [50]. Low consumption of n-3 PUFAs and excessive consumption ofn-6 PUFAs can worsen the inflammatory disease. On the other hand, human studies have found that the combination of increased consumption of n-3 PUFAs along with n- 6 PUFAs is associated with the lowest levels of inflammation in the body [56]. In addition, it was found that n-6 PUFAs do not inhibit the anti-inflammatory effects of n-3 PUFAs [56]. It has been thought that foods with higher ratios of n-3 to n-6 PUFAs (e.g., flaxseed oil or fish oil) can help decrease inflammatory disease processes [55].

\section{Summary and perspectives}

By 2025, the world is expected to have 300 million people with diabetes from 135 million in 1995 [57]. In 2012 in the United States alone, 30 million Americans had diabetes, 86 million Americans had prediabetes, and the total cost to treat diagnosed diabetes was around $\$ 245$ billion or about $\$ 7,900$ just to treat diabetes for one person [58]. It is also the 7th leading cause of death in America.

As discussed here, FA metabolism affects insulin actions in several ways in different cells and tissues. Some of them such as impairment of glucose usage and insulin action by FA in the muscle. On the other hand, the increase of PUFAs in the diet seems to attenuate insulin resistance at whole body level. With the growth of our understanding of FA and glucose metabolism and insulin signal transduction pathways in different cells and tissues, it only shows how complicate the impact of FA metabolism on insulin sensitivity could be. The observations that metabolism of FAs as a macronutrient affects insulin sensitivity provides targets of vulnerable pathways involved in insulin actions. The remaining questions become whether those pathways can only be influenced by FAs and how other factors such as micronutrients impact those pathways and in turn modulate insulin sensitivity. These are all significant questions that remain to be addressed.

From a public health point of view, we also need to consider how we can use the current knowledge to combat the epidemic of metabolic diseases such as the rapid increase of diabetic population globally. So, what can humans do to lower our chance of getting NIDDM and/or control it? Decreasing consumption of foods high in saturated fats (beef, pork, fried foods, etc.) and increasing consumption of foods high in MUFAs and PUFAs can help lower the prevalence of TAG in the blood stream and lower low density lipoprotein levels, which could result in lower chances of acquiring NIDDM [5]. In addition, having a low carb diet could also help lower blood glucose levels and decrease insulin resistance [2]. Dieting and exercise can help lower the chances of obesity and NIDDM to occur [39]. It has been shown that visceral fat can respond very well to diet and exercise, possibly resulting in less adipocyte insulin resistance [59].

There have been many advances in drugs can help treat and control NIDDM. Drugs such as thiazolidinedione can contribute to improving muscle and liver sensitivity to the hormone insulin [30]. Biguanides like metformin decrease hepatic gluconeogenesis, and sulfonylureas like glimepiride stimulate the pancreas to make more insulin over the course of a few hours post-prandial [60]. Fish and krill oils pills, which contain n-3 fatty acids, can also help decrease fatty acid impact by reducing TAG levels [61].

Future studies should attempt to look more closely at certain macronutrients such as different compositions of FAs or micronutrients such as vitamin A, which may impact the relationship between FA metabolism and insulin resistance [28]. Future studies should address the mechanisms by which PUFAs prevent the development of NIDDM.

\section{References}

1. Berg JM, Stryer L, Tymoczko JL. Stryer Biochemie: Springer-Verlag; 2015.

2. O'Sullivan TA, Hafekost K, Mitrou F, Lawrence D. Food sources of saturated fat and the association with mortality: a meta-analysis. American journal of public health. 2013;103(9):e31-e42. doi: 10.2105/ AJPH.2013.301492

3. Månsson HL. Fatty acids in bovine milk fat. Food \& nutrition research. 2008;52. doi: 10.3402/fnr.v52i0.1821

4. Jakobsen MU, O’Reilly EJ, Heitmann BL, Pereira MA, Bälter K, Fraser $\mathrm{GE}$, et al. Major types of dietary fat and risk of coronary heart disease: a pooled analysis of 11 cohort studies. The American journal of clinical nutrition. 2009;89(5):1425-1432. doi: 10.3945/ajcn.2008.27124 
5. Lunn J, Theobald $H$. The health effects of dietary unsaturated fatty acids. Nutrition Bulletin. 2006;31(3):178-224. DOI: 10.1111/j.14673010.2006.00571.x

6. van Meer G, de Kroon AI. Lipid map of the mammalian cell. J Cell Sci. 2011;124(Pt 1):5-8. doi: 10.1242/jcs.071233

7. Spector AA, Yorek MA. Membrane lipid composition and cellular function. Journal of lipid research. 1985;26(9):1015-1035.

8. Costanzo L. Fisiologia: Elsevier Brasil. 2014.

9. Mathews CK, Van Holde KE, Ahern KG. Bioquímica: Pearson Education. 2002.

10. Nelson DL, Lehninger AL, Cox MM. Lehninger principles of biochemistry: Macmillan. 2008.

11. Horowitz JF. Fatty acid mobilization from adipose tissue during exercise. Trends in Endocrinology \& Metabolism. 2003;14(8):386-392.

12. Faergeman NJ, Knudsen J. Role of long-chain fatty acyl-CoA esters in the regulation of metabolism and in cell signalling. Biochemical Journal. 1997;323(Pt 1):1-12.

13. Park S, Gammon S, Knippers J, Paulsen S, Rubink D, Winder W. Phosphorylation-activity relationships of AMPK and acetyl-CoA carboxylase in muscle. Journal of applied physiology. 2002;92(6):2475-2482.

14. Jakobsson A, Westerberg R, Jacobsson A. Fatty acid elongases in mammals: their regulation and roles in metabolism. Progress in lipid research. 2006;45(3):237-249.

15.Jump DB. Mammalian fatty acid elongases. Methods Mol Biol. 2009;579:375-389. doi: 10.1007/978-1-60761-322-0_19

16. Nakamura MT, Nara TY. Structure, function, and dietary regulation of $\Delta 6, \Delta 5$, and $\Delta 9$ desaturases. Annu Rev Nutr. 2004;24:345-376.

17. Control CfD, Prevention. National diabetes fact sheet: national estimates and general information on diabetes and prediabetes in the United States, 2011. Atlanta, GA: US Department of Health and Human Services, Centers for Disease Control and Prevention. 2011;201(1).

18. Cnop M, Welsh N, Jonas J-C, Jörns A, Lenzen S, Eizirik DL. Mechanisms of pancreatic $\beta$-cell death in type 1 and type 2 diabetes. Diabetes. 2005;54(suppl 2):S97-S107.

19. McGarry JD. What if Minkowski had been ageusic? An alternative angle on diabetes. SCIENCE-NEW YORK THEN WASHINGTON. 1992:258(5083):766-770.

20. Eizirik DL, Colli ML, Ortis F. The role of inflammation in insulitis and $\beta$-cell loss in type 1 diabetes. Nature Reviews Endocrinology. 2009;5(4):219-226. doi: 10.1038/nrendo.2009.21

21. Muoio DM, Newgard CB. Fatty Acid Oxidation and Insulin Action. Diabetes. 2008;57(6):1455-1456.

22. McGarry JD. Banting lecture 2001: dysregulation of fatty acid metabolism in the etiology of type 2 diabetes. Diabetes. 2002;51(1):7-18.

23. Boden G, Laakso M. Lipids and glucose in type 2 diabetes. Diabetes care. 2004;27(9):2253-2259.
24. Randle P, Garland P, Hales C, Newsholme E. The glucose fatty-acid cycle its role in insulin sensitivity and the metabolic disturbances of diabetes mellitus. The Lancet. 1963;281(7285):785-789.

25. Roden M. How free fatty acids inhibit glucose utilization in human skeletal muscle. Physiology. 2004;19(3):92-96.

26. Roden M, Price TB, Perseghin G, Petersen KF, Rothman DL, Cline GW, et al. Mechanism of free fatty acid-induced insulin resistance in humans. Journal of Clinical Investigation. 1996;97(12):2859.

27. McGarry JD, Mannaerts G, Foster DW. A possible role for malonyl-CoA in the regulation of hepatic fatty acid oxidation and ketogenesis. Journal of Clinical Investigation. 1977;60(1):265-270.

28. Chen G. The link between hepatic vitamin A metabolism and nonalcoholic fatty liver disease. Current drug targets. 2015;16(12):12811292.

29. Griffin ME, Marcucci MJ, Cline GW, Bell K, Barucci N, Lee D, et al. Free fatty acid-induced insulin resistance is associated with activation of protein kinase $C$ theta and alterations in the insulin signaling cascade. Diabetes. 1999;48(6):1270-1274.

30. Shulman GI. Cellular mechanisms of insulin resistance. The Journal of clinical investigation. 2000;106(2):171-176.

31. Itani SI, Ruderman NB, Schmieder F, Boden G. Lipid-induced insulin resistance in human muscle is associated with changes in diacylglycerol, protein kinase C, and IкB- $\alpha$. Diabetes. 2002;51(7):2005-2011.

32. Imamura F, Micha R, Wu JH, de Oliveira Otto MC, Otite FO, Abioye AI, et al. Effects of saturated fat, polyunsaturated fat, monounsaturated fat, and carbohydrate on glucose-insulin homeostasis: a systematic review and meta-analysis of randomised controlled feeding trials. PLoS Med. 2016;13(7):e1002087. doi: 10.1371/journal. pmed.1002087

33. Gastaldelli A, Toschi E, Pettiti M, Frascerra S, Quiñones-Galvan A, Sironi AM, et al. Effect of physiological hyperinsulinemia on gluconeogenesis in nondiabetic subjects and in type 2 diabetic patients. Diabetes. 2001;50(8):1807-1812.

34. Shulman GI. Ectopic fat in insulin resistance, dyslipidemia, and cardiometabolic disease. New England Journal of Medicine. 2014;371(12):1131-1141. doi: 10.1056/NEJMra1011035

35. Samuel VT, Petersen KF, Shulman GI. Lipid-induced insulin resistance: unravelling the mechanism. The Lancet. 2010;375(9733):2267-2277. doi: 10.1016/S0140-6736(10)60408-4

36.Savage DB, Petersen KF, Shulman GI. Disordered lipid metabolism and the pathogenesis of insulin resistance. Physiological reviews. 2007;87(2):507-520.

37. Martins AR, Nachbar RT, Gorjao R, Vinolo MA, Festuccia WT, Lambertucci $\mathrm{RH}$, et al. Mechanisms underlying skeletal muscle insulin resistance induced by fatty acids: importance of the mitochondrial function. Lipids in health and disease. 2012;11(1):30. doi: 10.1186/1476-511X- 


\section{$11-30$}

38. Anderson CM, Stahl A. SLC27 fatty acid transport proteins. Molecular aspects of medicine. 2013;34(2-3):516-528. doi: 10.1016/j. mam.2012.07.010

39. Maggio CA, Pi-Sunyer FX. Obesity and type 2 diabetes. Endocrinology and metabolism clinics of North America. 2003;32(4):805-822.

40. Arner P. Free fatty acids-do they play a central role in type 2 diabetes? Diabetes, Obesity and Metabolism. 2001;3(s1):11-19.

41. Kissebah AH, Krakower GR. Regional adiposity and morbidity. Physiological reviews. 1994;74(4):761-811.

42. Guilherme A, Virbasius JV, Puri V, Czech MP. Adipocyte dysfunctions linking obesity to insulin resistance and type 2 diabetes. Nature reviews Molecular cell biology. 2008;9(5):367-377. doi: 10.1038/ nrm2391

43. Psychic S. Fats, Oils, Fatty Acids. Triglycerides-Chemical Structure. 2013;16.

44. Riccardi G, Giacco R, Rivellese A. Dietary fat, insulin sensitivity and the metabolic syndrome. Clinical nutrition. 2004;23(4):447-456.

45. Vessby B, Uusitupa M, Hermansen K, Riccardi G, Rivellese AA, Tapsell LC, et al. Substituting dietary saturated for monounsaturated fat impairs insulin sensitivity in healthy men and women: The KANWU Study. Diabetologia. 2001;44(3):312-319.

46. Hodge AM, English DR, O'dea K, Sinclair AJ, Makrides M, Gibson RA, et al. Plasma phospholipid and dietary fatty acids as predictors of type 2 diabetes: interpreting the role of linoleic acid. The American journal of clinical nutrition. 2007;86(1):189-197.

47. Bang H, Dyerberg J, Sinclair HM. The composition of the Eskimo food in north western Greenland. The American journal of clinical nutrition. 1980;33(12):2657-2661.

48. Lee JS, Pinnamaneni SK, Eo SJ, Cho IH, Pyo JH, Kim CK, et al. Saturated, but not n-6 polyunsaturated, fatty acids induce insulin resistance: role of intramuscular accumulation of lipid metabolites. Journal of applied physiology. 2006;100(5):1467-1474.

49. Cavaliere G, Trinchese G, Bergamo P, De Filippo C, Raso GM, Gifuni G, et al. Polyunsaturated fatty acids attenuate diet induced obesity and insulin resistance, modulating mitochondrial respiratory uncoupling in rat skeletal muscle. PloS one. 2016;11(2):e0149033. doi: 10.1371/ journal.pone.0149033

50. Calder PC. Polyunsaturated fatty acids, inflammation, and immunity. Lipids. 2001;36(9):1007-1024.

51. Johnson GH, Fritsche K. Effect of dietary linoleic acid on markers of inflammation in healthy persons: a systematic review of randomized controlled trials. Journal of the Academy of Nutrition and Dietetics. 2012;112(7):1029-1041. doi: 10.1016/j.jand.2012.03.029

52. Fritsche KL. The science of fatty acids and inflammation. Advances in Nutrition: An International Review Journal. 2015;6(3):293S-301S. doi: 10.3945/an.114.006940

53. Sekiya M, Yahagi N, Matsuzaka T, Najima Y, Nakakuki M, Nagai R, et al. Polyunsaturated fatty acids ameliorate hepatic steatosis in obese mice by SREBP-1 suppression. Hepatology. 2003;38(6):1529-1539.

54. Calder PC. Omega-3 fatty acids and inflammatory processes. Nutrients. 2010;2(3):355-374. doi: 10.3390/nu2030355

55. James MJ, Gibson RA, Cleland LG. Dietary polyunsaturated fatty acids and inflammatory mediator production. The American journal of clinical nutrition. 2000;71(1):343s-348s.

56. Pischon T, Hankinson SE, Hotamisligil GS, Rifai N, Willett WC, Rimm EB. Habitual dietary intake of n-3 and n- 6 fatty acids in relation to inflammatory markers among US men and women. Circulation. 2003;108(2):155-160.

57. King H, Aubert RE, Herman WH. Global burden of diabetes, 19952025: prevalence, numerical estimates, and projections. Diabetes care. 1998;21(9):1414-1431.

58. Anderson H. Getting Real About Food:" Fed Up" \& Nutrition Education. 2015.

59. Yamauchi T, Kamon J, Waki H, Terauchi Y, Kubota N, Hara K, et al. The fat-derived hormone adiponectin reverses insulin resistance associated with both lipoatrophy and obesity. Nature medicine. 2001;7(8):941-946.

60. Center JD. Oral diabetes medications summary chart. 2013.

61. Friedberg CE, Janssen MJ, Heine RJ, Grobbee DE. Fish oil and glycemic control in diabetes: a meta-analysis. Diabetes care. 1998;21(4):494500 . 